\title{
Development and Evaluation of the Binary Hologram Master
}

\author{
Yoichi Higuchi \\ Intellectual property, Dai Nippon Printing Co. Ltd. \\ 1-1-1 Kagamachi, Ichigaya, Shinjyuku, Tokyo 162-8001, Japan.
}

\begin{abstract}
The binary hologram master with two-layer refractive index was developed and evaluated of their characteristics. The chirped diffraction grating was fabricating by electron-beam lithography. The grating structure has a one-dimensional grating with a period of $0.26 \mu \mathrm{m}$. The polarization separation lenses consist of two-layer, substrate materials and coated on thin-film materials. Example designs are presented, and the conventional diffraction analysis is used to evaluate the performance of these gratings. As an example, measurement of two-layer film is carried out and excellent confirmation is obtained.
\end{abstract}

Keywords: electron beam lithography, refractive index, polarization separation element, hologram master

\section{Introduction}

Electron-beam (EB) lithography has proved to be excellent for manufacturing diffractive optics surface [1]. The holographic grating based on both the surface relief structure and refractive index modulation has potential applicability for optical elements and devices [2]. It was recently predicted that highly efficient diffractive optical elements with unique properties could be realized by fabrication of surface-relief structures with features smaller than the wavelength of light in the material [3]. The proposed diffractive structures are binary phase gratings with grooves of varying width and spacing depth [4]. The lateral dimensions of the individual ridges and grooves of the grating are smaller than the wavelength of light in the respective materials. The grating structure has the properties of a homogeneous layer of material with an effective index of refraction determined by the duty cycle of the features at the grating surface. Higher diffraction orders emerge from a grating at an angle. If the grating period is extremely small, the light becomes evanescent. Diffraction optical devices can be realized that use the variations in the grating dimensions to produce gradient effective index-of refraction surfaces with very high diffraction efficiencies.

The intensity and phase of the transmitted or reflected light from a zero-order grating depend on polarization. Thin film antireflection coatings are often used to produce low reflectivity [5]. Thin films degrade at high power and have a limited range of indices of reflection, the minimum being $\sim 1.35$ [6]. Alternatively a high-spatial frequency surface-relief binary grating on a substrate can also behave as an antireflection surface.

The author report high efficiency surface-relief grating fabricated for hologram color filters. Gratings can be fabricated out of cheaper materials, and replication technology is available for mass production. In this paper the author design binary gratings with two-layer reflective index, the effective refractive index of a microstructure is simulated and fabricated for distributed-index structures based on zeroorder gratings with variable duty cycle are proposed. The author 
investigates an adequate cell size, and fabricates the chirped-period grating according to result of the proximity correction. The physical principle of artificial distributed-index mask characterized as a compositional two-layer structure is demonstrated.

\section{Experimental}

2.1 Materials and Measurements.

ZEP-520 is the EB positive resist supplied by Nippon Zeon Co.Ltd. The holographic recording material for Omnidex 352 is a panchromatic photopolymer supplied by Dupont. Holograms are recorded on the photopolymer using an argon ion laser beam $(514.5 \mathrm{~nm})$ at an output $5 \mathrm{~W}$. After recording, the photopolymer is exported to ultraviolet (UV) light. A $50 \mathrm{KeV} \mathrm{EB}$ exposure system (Hitachi) was used in the evaluation of the resist performance such as sensitivity and resolution capability. The resist patterns were observed with a scanning electron microscope (SEM). A profiler Dektak8000 (Sloan) was used for measurement of the film thickness of resist after development. The completed mask was inspected with a HRL219-PS (KLA). Fig. 1 shows the schematic diagram of the experimental setup for the holographic grating and observing the intensity of the diffracted beam. A nonpolarized $\mathrm{He}-\mathrm{Cd}$ laser beam was used a probing beam. A $\mathrm{He}-\mathrm{Cd}$ laser beam is split using the beam splitter (BS). The two beams, after reflection from the mirror (M1, M2) are allowed to interfere with each other at the holographic grating. Two polarizes are used to control the polarization state of the measuring beams. The power density of the beams was $2.7 \mathrm{~mW} / \mathrm{cm}^{2}$ and incident angle $\theta$ was selected. The diffraction efficiency of the +1 st order diffracted beam from the gratings in transmission mode was monitored as a function of time using an optical

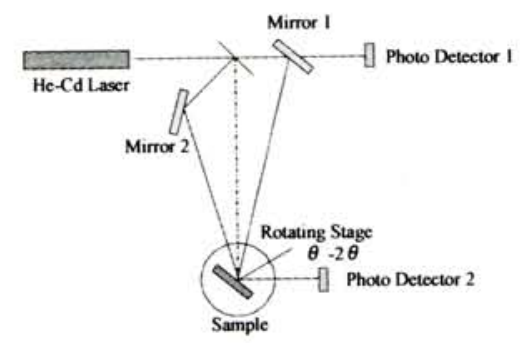

Fig.1 Schematic diagram of the measurement apparatus powermeter (Newport, 1815-C).

\subsection{Design of the hologram master}

Fig. 2 shows schematically the profile of a rectangular surface-relief grating with period $r$, grating depth $\mathrm{h}$, and duty cycle $\mathrm{f}$. It is composed of two materials with refractive indices $\mathrm{n} 1$ and $\mathrm{n} 2$. Light with a wavelength $\lambda$ will not be diffracted into diffraction orders outside the grating.

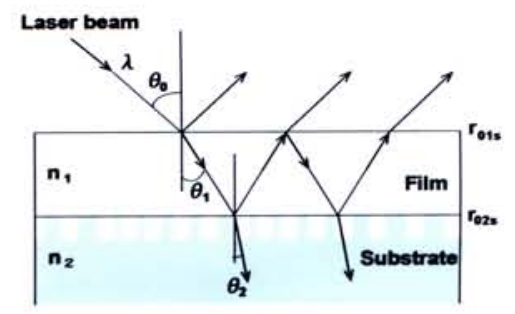

Fig.2 Schematic diagram of reflection from the transparent thin films on a surface-relief grating.

In a crude approximation the electric field within the grating grooves is considered homogeneous. Then the grating behaves like a negative uniaxial and extraordinary beams follows from the birefringence,

$$
\mathrm{n}_{\mathrm{TE}}=\sqrt{(1-\mathrm{f}) \mathrm{n}_{1}^{2}+\mathrm{fn}_{2}^{2}} n_{\mathrm{TM}}=\sqrt{\frac{\mathrm{N}_{1}^{2} \mathrm{n}_{2}^{2}}{\mathrm{fn}_{1}^{2}+(1-\mathrm{f}) \mathrm{n}_{2}^{2}}}
$$

(Where f denotes ratio of land area to period $r$ ) [7]. Physically, different effective refractive index for each polarization mode means that when light passes through a structure with period smaller than the light wavelength, the structure is regarded as a material that generates scattering. If the period has the same order of magnitude as the wavelength, the band edge for waves in a periodic material influences the effective index. The procedure presented is applicable for both TE and TM polarization and for any angle of incidence. Specifically a polarization-selective mirror designed to be antireflective for TE or TM incident polarization and maximum reflectivity for the orthogonal polarization TM by the appropriate selection of a grating. The filling factor and the groove depth of the grating calculated in the long-wavelength limit to obtain zero reflectivity for one polarization and maximum reflectivity for the orthogonal polarization. Thus the binary grating exhibits the properties of a polarization-selective mirror. An exact method, the rigorous coupled-wavelength analysis of surface relief 
gratings, is used to verify the selective antireflection behavior of the designed gratings.

\subsection{Principle of the HCF}

HCF (Hologram Color Filter) is a HOE that separates white light into primary colors [8]. Hologram lens elements are located in front of reflecting electrode at a distance of just focal length of the hologram lens. The center pitch is as fine as $0.41 \mu \mathrm{m}$. White light from a light source is converted TM (or TE) by a polarizing device, and impinges on HCF at a slant angle. This beam is focused and dispersed through the diffraction of the $\mathrm{HCF}$, so that a color spectrum pattern is generated on the plane of liquid crystal. The light beam is converted by reflecting electrode. The reflected beam impinges on the HCF again.

Second, re-entrance light impinges on a different point from a first pass point in the HCF plane, and consequently doesn't match Bragg's condition. After passing a polarizer, the image is enlarged and projected to a screen by projection lens. The production process is composed of 4 steps that is design of a grating pattern, making of a binary hologram master, making of a volume hologram master and contract printing to a final hologram [9].

\section{Results and Discussion}

3.1 Design of the binary gratings and angle of incident

The first task is to design a surface-relief binary grating that efficiently couples with light into the first diffracted order. The Polyvinylalcohol (PVA) film was coated with thickness of $1000 \AA$ on a binary hologram mask (quartz) and soft baked by heating on a hot-plate $\left(80-85^{\circ} \mathrm{C}\right)$. The film sample of the PVA was contained $2.5 \%$ concentration of the Red-dye (Anthoraqunone derivative). This corresponds to a complex effective refractive index to Bragg reflection at the grating. For crossed gratings both polarization experience the same effective refractive index [10]. The effective refractive index depends on the duty cycle. Thus a grating with variable duty cycle behaves like an artificial distributed index medium. A brazed grating requires that within one period of zero-grating the phase of the light changes linearly from 0 to $2 \pi$. The diffraction efficiency was calculated for the grating substrate. Because the index of refraction of the substrate is much higher than the index of refraction of air, the author expect more propagating orders in the refraction angle, the 1st order not vanish even when the incident angle reaches $80^{\circ}$. The grating equation can be written as

$$
\operatorname{Sin}\left(\theta_{\mathrm{t}}\right)=\sin \left(\theta_{\mathrm{i}}\right)+(\mathrm{m} \lambda / \Lambda)
$$

Where $\theta_{\mathrm{i}}$ is the incident angle and $\theta_{\mathrm{t}}$ is the diffraction angle for the $\mathrm{m}$-Th order [11]. Both are the angle in air. According to this equation, the 1st order became evanescent when the incident angle reaches $64^{\circ}$. The value of the reflective film index was obtained by using the reflectance data at $55.0^{\circ}$ and $70.0^{\circ}$, which were fairly distant from the angle of $\theta$. As the result, the differences between the theoretical and measured reflectance of 0.08917 at $62^{\circ}$ were estimated. This corresponds to the introduction of an experimental error of $1 \%$ for measuring the reflectance. Fig. 3 shows relation between angle of incidence and reflection ratio. For different incident angles, the optical path inside the antireflection layer, and therefore the effective layer thickness, will be different. In the above design method, the grating form is determined without considering the reflection of light at the top and bottom boundaries of the subwavelength grating film.

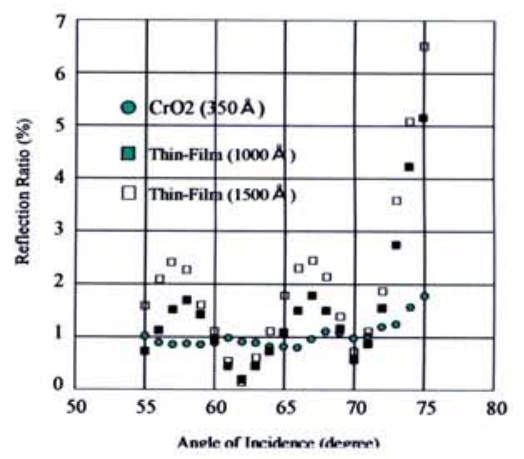

Fig.3 Dependence of the reflectance on an angle of incidence.

The binary hologram master is made from the grating pattern data. The grating pattern data is generated by computer, like ordinary computer generated hologram (CGH). This grating is composed of linear line and space and has gradation pitch for the cylindrical focusing function. The diffraction grating lens(a cylindrical lens) was 280 $\mu \mathrm{m}$ in width, and its focal length was $1000 \mu \mathrm{m}$. 
The maximum grating period was $750 \mathrm{~nm}$ at the middle point of the gratings, and the minimum period was $150 \mathrm{~nm}$ at the grating ends.

3.2 Development of the binary hologram master (EB lithography)

The author fabricated the chirped-period diffraction grating according to the calculated electron dosage. The electron dosage was modulated by 32-64 levels, which depend on the grating periods. It is found that the subfield boundaries were removed by adjusting the subfield size including the proximity of the EB writing. The optimum distributions depended on the grating period because of the proximity of the EB exposure.

The mask IP is measured with an IP metrology tool, Leica LMS-IPRO. First the IP of the resist image is measured after $\mathrm{EB}$ writing. IP marks are placed with $250 \mu \mathrm{m}$ pitch for X-directions and Y-directions. The hole in each die is $250 \mathrm{~nm}$ square and its array X-pitch /Ypitch is $300 \mathrm{~nm}$. Experiment and simulation are compared using the displacements at the centers of the four edges. The IP maps of experiment are show in Fig. 4.

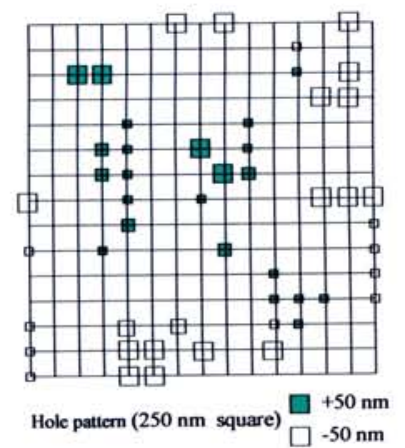

Fig.4 Experimental results for the test pattern of $250 \mathrm{~nm}$ hole in width.

The displacement at each measurement point on a grid is represented. Displacements predicted by simulation are found to be smaller than experimental values. The mask requirement on CD uniformity of less than or equal to $0.05 \mu \mathrm{m}$ was satisfied by EB process and Developing process.

A scanning electron micrograph of a part of the fabricated grating is shown in Fig. 5. The grating has good appearance for lens. The measured grating profile agrees well with the estimated profile. The gratings were designed to have a groove depth of $0.35 \mu \mathrm{m}$ and to be 150-270

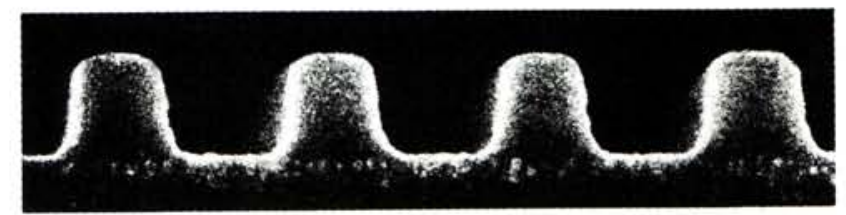

Fig.5 Scanning electron micrograph of grating pattern (Cross section).

$250 \mathrm{~nm}$

$\mathrm{nm}$ in size. It is found that the fabricated grating structure has a smooth surface, a sharp edge with an almost right $87^{\circ}$ angle, and qualities that are necessary to achieve high efficiency.

\subsection{Evaluation of the hologram master and HCF}

As a first in evaluating the performance of reflective thin-film, calculated the retardation and transmittance for the structure [12]. The phase retardation as a function of wavelength is shown in Fig. 6(a) and the transmittance of TE and TM wave is shown in Fig. 6(b). In the wavelength

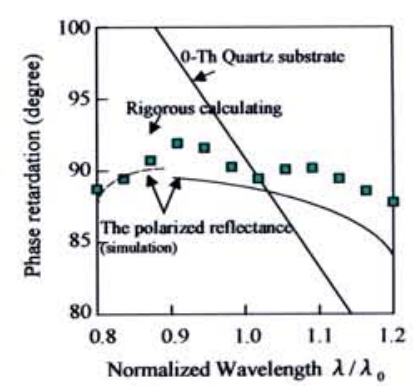

(a) Phase retardation

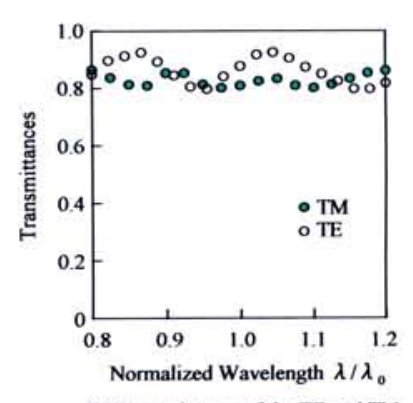

(b)Transmittance of the TE and TM
Fig.6 Performance of the surface-relief grating: (a) Phase of the retardation (b) Transmittance of the TE and TM wave with respect to wavelength.

range from $0.8 \lambda_{0}$ to $1.0 \lambda_{0}$, the retardation varies between the transmittance of TM and TE is $17 \%-22 \%$. The variation in retardation and difference in transmittances are so large that the subwavelength grating cannot use, as the hologram master can't. The undulation of the retardation and transmittance curves is caused by light reflection at the top and bottom boundaries of the grating structure. The two-layer reflective index structure reduces the light reflection at both boundaries. The thin-film set the refractive indices of the upper and lower mediums at 1.58 because the effective refractive index of the TE wave is 1.57 at the normal wavelength $\lambda_{0}$. The most effective index of TM wave is 1.55 at $\lambda_{0}$. For the transmittance curve of a TM wave, a small amount of undulation remains. Generally hologram can be made by two 
methods. The one is relief phase hologram and another is amplitude hologram. The amplitude hologram is adopted into this HCF because index-matching fluid must be used at the contact copy process. If relief type is used, the index matching fluid fills up the groove of the relief, and eliminates the diffraction effect.

The binary hologram master has low light efficiency, which is sum of 0th order and 1st order efficiency, as an ordinary amplitude hologram does. If this is used for a contract copy process, long exposure time is needed and the production yield gets low. The volume hologram master for massproduction process was made, which has higher light efficiency. The volume hologram master is made by contact print method from the binary hologram master. To get a final hologram with high diffraction efficiency, Oth order beam and 1st order beam of the volume hologram master must have approximately same diffraction efficiency. The diffraction efficiency (DE) of the volume hologram is $100 \%$ theoretically and the optical efficiency in each color has been improved.

Table 1 shown samples of diffraction efficiency of the binary hologram master, the volume hologram master and the final hologram. In the

Table 1 Diffraction efficiency of grating hologram master and final hologram.

\begin{tabular}{|l|c|c|}
\hline \multirow{2}{*}{} & \multicolumn{2}{|c|}{ Diffraction Efficiency (\%) } \\
\cline { 2 - 3 } & Oth order & 1st order \\
\hline Binary hologram master & $<10$ & $<10$ \\
\hline Volume hologram master & $35-45$ & $40-50$ \\
\hline Final hologram (HCF) & $5-10$ & $87-93$ \\
\hline
\end{tabular}

hologram master, 0th order beam and 1st order beam have approximately same diffraction efficiency. Final hologram's efficiency is 87 to $93 \%$. As a result, the superior and optical characteristic imaging was performed.

\section{Conclusion}

The design of the binary hologram master which have predicted diffraction efficiencies about 93\%. The binary grating was surprisingly insensitive to the polarization and angle of incident light. The grating profiles utilized as a binary hologram master was evaluated by regarding the 1st-order and 2nd-order diffracted light. Grating depth $0.27 \mu \mathrm{m}$ of duty ratio 1.52 was achieved in the maximum $\mathrm{DE}$ of the $\mathrm{HCF}$.

The experimental results show that this grating master provides high measured polarization extinction ratios. Printing the hologram color filter has also been shown to have the capabilities for operating with optical signals of wide angular bandwidth with a high extinction ratio (170:1) and significant efficiencies unless $87 \%$. The mass production of such HOEs would be possible by the development of a precise replication technology from EB lithographed elements. These characteristics make the devices desirable for use in image processing, and in many other polarization optics applications.

\section{Acknowledgements}

The author thanks the electronic device laboratory at Dai Nippon Printing (DNP), where this work was performed.

\section{References}

1. M. Ekberg, F. Nikolajeff, M. Larsson, and S. Hard: Appl. Opt., 33, (1994) 103.

2. J. F. Johnson, G. W. Kammlott and K. A. Ingersoll: Appl. Opt., 17, (1978) 1165.

3. J. M. dos Santos and L. M. Bernardo: Appl. Opt., 36, (1997) 8935.

4. M. W. Farn: Appl. Opt., 31, (1992) 4453.

5. M. Chang, U. J. Gibson: Appl. Opt., 24, (1985) 504.

6. L. H. Cesccato, E. Gluch, and N. Streibl: Appl. Opt., 29, (1990) 3286.

7. M. Born and E. Wolf Principles of Optics, Pergamon, New York (1980) 707.

8. R. D. Sterling and W. P. Bleha: $I W 97$, (1997) 809.

9. Y. Higuchi, T. Segawa, M. Kurihara, and D. Totsukawa: J. Photopolym. Sci. Technol., 18, (2005) 175.

10. D. E. Aspnes: J. Vac. Sci. Techonl, 18, (1981) 289.

11. A. H. M. Holtslag and P. M. L. O. Scholte: Appl. Opt., 28, (1989) 5095.

12. D. M. Tennant, K. F. Dreyer, K. Feder, R. P. Gnall, T. L. Koch, U. Koren, B. I. Miller, C. Vartuli, and M. G. Young: J. Vac. Sci. Techonl., 12, (1994) 3689 . 